\title{
Spinal cord injury and its underlying mechanism in rats with temporal lobe epilepsy
}

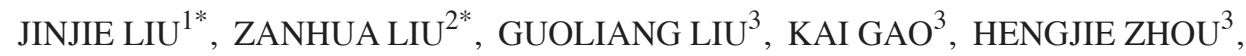 \\ YONGBO ZHAO $^{4}$, HONG WANG ${ }^{1}$, LIN ZHANG ${ }^{5}$ and SIBO LIU $^{3}$ \\ ${ }^{1}$ General Ward II, Affiliated Municipal Central Hospital of Dalian Medical University, Dalian, Liaoning 116000; \\ ${ }^{2}$ Department of Neurology, Nanjing Gaochun People's Hospital, Gaochun, Nanjing 210000; ${ }^{3}$ Surgical Intensive Care Unit, \\ Dalian Municipal Central Hospital, Dalian, Liaoning 116000; ${ }^{4}$ Department of Neurology, The First People's \\ Hospital of Shanghai Jiaotong University, Shanghai 200000; ${ }^{5}$ Department of Neurology, Affiliated Municipal \\ Central Hospital of Dalian Medical University, Dalian, Liaoning 116000, P.R. China
}

Received March 28, 2019; Accepted November 29, 2019

DOI: $10.3892 /$ etm.2020.8453

\begin{abstract}
Numerous cases of spinal cord injury following seizure have been previously reported. However, whether spinal cord injury is a common occurrence after seizures and its underlying mechanisms remain unclear. The present study generated a Sprague-Dawley rat model of temporal lobe epilepsy (TLE), and Nissl staining and transmission electron microscopy were used to detect tissue damage. In addition, Evans blue staining was used to detect damage to the blood-brain barrier (BBB) and albumin extravasation. In addition, double-staining was used to detect the association between neurons and extravasated albumin. Furthermore, neuronal degeneration was assessed using Fluoro-Jade C staining, while fluorescence staining and western blotting were used to detect apoptosis and inflammation. In the present study, spinal cord injury was only observed in rats with grade IV-V seizures, whereas Nissl staining showed structural damage and decreased neuronal cell numbers in the brain and the spinal cord. The present study identified BBB damage and albumin extravasation in rats of the TLE groups. Double-staining for albumin and neurons showed a significant match of neurons positive for albumin. Fluoro-Jade $\mathrm{C}$ staining indicated neuronal degeneration in the brain, but not the spinal cord in the TLE rats. High levels of caspase- 3 were also detected in the injured spinal cord. A small number of albumin ${ }^{+}$neurons in the spinal cord presented caspase $-3^{+}$signals in rats of the
\end{abstract}

Correspondence to: Dr Sibo Liu, Surgical Intensive Care Unit, Dalian Municipal Central Hospital, 826 Xinan Road, Dalian, Liaoning 116000, P.R. China

E-mail: icu2000@163.com

${ }^{*}$ Contributed equally

Key words: temporal lobe epilepsy, seizure, spinal cord injury, brain blood barrier, albumin, inflammation
TLE groups. The expression levels of intercellular adhesion molecule 1, CD11b and inflammatory factors such as tumor necrosis factor- $\alpha$ and interleukin- 6 were significantly elevated in the injured spinal cord. The present results suggested that spinal cord injury occurred in rats as a result of severe seizure attacks, and that BBB damage, albumin extravasation, inflammation and apoptosis contributed to the pathological changes observed during spinal cord injury.

\section{Introduction}

Several clinical cases of spinal cord injury following seizure have been reported (1-3), including those in primary (1) and secondary epilepsy (3). In grand mal epilepsy, impairments of the face and head commonly occur due to mechanical injury (4). Spinal cord injury is also considered to be caused by trauma or strong muscular contractions $(2,3)$, especially in severe refractory epilepsy $(2,3)$. The theory of mechanical damage is also supported by a high incidence of cervical-spinal cord injury in walking adults with severe refractory epilepsy accompanied by falls and other injuries, especially head injury (3). Thoracic and lumbar injury have also been reported and are considered to be caused by strong muscular contractions during seizures $(2,3)$. However, there are some limitations to the mechanical injury hypothesis, as not all patients with spinal cord injury show evidence of trauma or other underlying lesions (1). Furthermore, the nature of the pathological changes following epilepsy-associated spinal cord injury are not fully understood. A previous study demonstrated degeneration of the spinal cord in myoclonus epilepsy (5). Another previous study showed that after seizure, the expression levels of serum C-response protein, cytokines and inflammatory factors are markedly increased, thus indicating a systemic seizure-associated inflammatory reaction (6). The brain and spinal cord share a common origin, which is supported by the co-expression of specific neurotransmitters in both locations $(7,8)$. Therefore, there may be a close connection between spinal cord injury and the abnormal discharge of brain neurons $(7,8)$. 
Previous studies related to spinal cord injury after epileptic seizure are relatively limited, and whether spinal cord injury commonly occurs following epileptic seizures is unclear. In addition, the mechanism underlying epilepsies-mediated injury remains to be investigated. Our previous study investigated severe neuronal injury, damage to the blood-brain barrier (BBB) and neuronal uptake of serum albumin in the brain parenchyma of a kainic acid (KA)-induced rat model of temporal lobe epilepsy (TLE) $(7,8)$. The present study aimed to investigate the pathological changes and underlying mechanisms of spinal cord injury in TLE. Sprague-Dawley rats were intraperitoneally injected with pilocarpine (PL; PL group) or stereotaxically administered KA (KA group) to rule out the possibility of spinal cord injury caused by cerebrospinal leakage of KA via hippocampal directional injection. These groups were used to investigate the correlation between spinal cord injury and epileptic seizures in two different models.

\section{Materials and methods}

Ethical approval and animal preparation. The present study was approved by The Ethics Committee of The First People's Hospital of Shanghai Jiaotong University. All experimental procedures were performed according to the Laboratory Animal Care standards of The Ethics Committee, and all efforts were made to minimize the number of animals used and the level of suffering to those involved. Male Sprague-Dawley rats (total n=90; weight, 250-300 g; age, 8-9 weeks old; Shanghai SIPPR-Bk Lab Animal Co., Ltd.) were independently raised in plastic cages with a 12 -h light/dark cycle at $20-25^{\circ} \mathrm{C}$ with relative humidity 50-65\% and ventilation 8-12 times/h, and free access to food and water. The rats were divided into three groups: i) The control group that had a stereotactic $(n=15)$ or intraperitoneal injection $(n=15)$ of saline, total $n=30$; ii) the KA group that had a stereotactic injection of KA, $n=30$; and iii) the PL group that had an intraperitoneal injection of PL, $n=30$.

Model of TLE and electrode implantation. The rats were anesthetized with an intraperitoneal injection of $350 \mathrm{mg} / \mathrm{kg}$ $10 \%$ chloral hydrate (Sigma-Aldrich; Merck KGaA). Local disinfection using an alcohol cotton ball and aseptic surgery were conducted to avoid infection. There were no significant signs of peritonitis, such as fever, abdominal muscle tension or abdominal tenderness. Following anesthesia, the rats were fixed onto stereotaxic apparatus (Model UMC4; World Precision Instruments, Inc.). The fonticulus anterior, sagittal suture and fonticulus posterior were exposed and adjusted to the same horizontal position. A microchannel (coordinates with the Bregma: Anteroposterior, $-3.6 \mathrm{~mm}$; mediolateral, $-3.4 \mathrm{~mm}$; and dorsoventral, $-3.8 \mathrm{~mm}$ ) was established in the skull for the intra-hippocampal administration of $0.5 \mathrm{mM} \times 1 \mu 1,0.05 \mu \mathrm{l} / \mathrm{min} \mathrm{KA}$. Preliminary tests were performed to determine the optimal dosage of KA for triggering an epileptic seizure; $0.5 \mathrm{mM} \times 1 \mu 1,0.05 \mu \mathrm{l} / \mathrm{min}$, as previously described $(7,8)$. Rats in the control group were injected with $1 \mu \mathrm{l}$ sterile saline. The rats in the PL group received an intraperitoneal injection of $350 \mathrm{mg} / \mathrm{kg}$ PL $(0.5 \mu \mathrm{l})$, and the control group received an equal volume of saline. Deep-recording electrodes (Electroencephalograph Analysis system; version 1.70; Cadwell Industries, Inc.) were implanted into the bilateral hippocampal Cornu Ammonis 3 region using the aforementioned coordinates. A monopolar electrode was implanted into the bone above the cerebellum. All electrodes were fixed to the skull using screws and embedded with dental cement (Paladur; Kulzer $\mathrm{GmbH})(7,8)$. To generate a clear electroencephalograph (EEG) image, a high-resolution recorder (EEG-1200C; Nihon Kohden Corporation) was connected. Immediately after surgery, both EEG recording and behavioral video monitoring were conducted for $12 \mathrm{~h}$. Behavioral characteristics were evaluated by an independent researcher who was blinded to the experiment using video recording according to Racine's V-point Scale (9), as follows: i) I, movement of the mouth, lips, tongue and vibrissae; ii) II, head clonus; iii) III, forelimb clonus, frequent and rhythm head and neck shaking; iv) IV, clonic rearing; and v) $\mathrm{V}$, uncontrolled jumping and clonic rearing with loss of postural control.

Sampling. According to our previous study, pathological changes in the brain were most significant between days 3 and 7 post-injection of KA $(10,11)$; therefore, all rats were sacrificed on the 3rd day after TLE induction. The rats were individually anesthetized with an intraperitoneal injection of $350 \mathrm{mg} / \mathrm{kg}$ $10 \%$ chloral hydrate, and sacrificed via cardiac perfusion with $70 \mathrm{ml}$ warmed saline and $40 \mathrm{ml} 4 \%$ paraformaldehyde (PFA; Sigma-Aldrich; Merck KGaA; $4^{\circ} \mathrm{C} ; \mathrm{pH} 7.4 ; 0.1 \mathrm{M} ; 100 \mathrm{ml} / \mathrm{each}$ ) when in a state of deep anesthesia. Death was confirmed when respiration and a heartbeat were undetectable, and the pupils were completely dilated. The intact brain and spinal cord, including the cervical cord, thoracic cord and lumbar cord, were excised and submerged in $4 \% \mathrm{PFA}$ at $4^{\circ} \mathrm{C}$ for $18 \mathrm{~h}$. The brain and spinal cord were then dehydrated with $30 \%$ sucrose in $0.9 \%$ normal saline ( $\mathrm{pH} 7.4)$ at $4^{\circ} \mathrm{C}$ for 3 days; the sucrose was replaced once a day to ensure efficient dehydration. Samples were dried and embedded in Optimum Cutting Temperature compound (OCT, cat. no. 4583; Leica Microsystems GmbH), and stored at $-80^{\circ} \mathrm{C}$ prior to immunoassay analysis. For transmission electron microscopy (TEM), the brain and spinal cord samples were fixed with $1.3 \%$ glutaraldehyde at $4^{\circ} \mathrm{C}$ for $3 \mathrm{~h}$ immediately after excision.

Nissl staining for the detection of neuronal injury. Nissl staining was performed to detect neuronal injury. After fixed with $4 \%$ PFA at $4^{\circ} \mathrm{C}$ for $18 \mathrm{~h}$ and embedded using OCT at $-80^{\circ} \mathrm{C}$, sections of the brain and spinal cord were cut into $35 \mu \mathrm{m}$ slices using a sliding microtome (CM1950; Leica Microsystems $\mathrm{GmbH})$. After washing with $\mathrm{PBS}$ at $\mathrm{pH} 7.4$, the tissues were dried at $55^{\circ} \mathrm{C}$ for $3 \mathrm{~h}$ and immersed in $0.9 \%$ crystal violet at $37^{\circ} \mathrm{C}$ (Sigma-Aldrich; Merck KGaA) for $2 \mathrm{~h}$. The tissues were dehydrated with 70, 80, 90 and $100 \%$ ethanol for $5 \mathrm{~min}$, and mounted with neutral balsam. Observations were performed using a fluorescent microscope (100 $\mu \mathrm{m}$; Eclipse 80i; Nikon Corporation). ImageJ software (version 1.42q; National Institutes of Health) was used to conduct densitometric analysis and to determine the mean analysis area of $\mathrm{Nissl}^{+}$cells. An average value was obtained by observing six random fields from each of the ten slices per rat.

TEM. The brains, primarily the hippocampus and the cortex, and the cervical, thoracic and lumbar spinal cord from all 
groups were removed and fixed with $1.3 \%$ glutaraldehyde at $4^{\circ} \mathrm{C}$ for $3 \mathrm{~h}$ before submission. After embedded with epoxy resin at $60^{\circ} \mathrm{C}$ for $36 \mathrm{~h}$, samples were cut into $0.06 \mu \mathrm{m}$ sections and stained with $0.5 \%$ uranyl acetate at $4^{\circ} \mathrm{C}$ for $1 \mathrm{~h}$. Structural damage of the neuronal tissue and $\mathrm{BBB}$ was observed using a transmission electron microscope (Scale bars $0.5 \mu \mathrm{m}, 1 \mu \mathrm{m}$; JEM-1230; JEOL, Ltd.).

Measurement of brain and spinal cord edema. The degree of brain and spinal cord edema in TLE was determined using the wet-dry weight method (12). The wet weight of the tissues was obtained immediately after dissection and the dry weight was measured after drying at $55^{\circ} \mathrm{C}$ for $24 \mathrm{~h}$. The water content and degree of tissue swelling was calculated as follows: Water content $(\%)=($ wet weight-dry weight) $\mathrm{x} 100 /$ wet weight.

Double immunofluorescence staining of albumin and neuronal cells. To determine the integrity of the BBB and the distribution of serum albumin, double-staining of albumin and neuronal cells was conducted. The rats were injected with $4 \mathrm{ml} / \mathrm{kg} \mathrm{2 \%}$ Evans blue (EB), which directly binds to serum albumin, via the tail vein $4 \mathrm{~h}$ before transcardiac perfusion. EB administration was performed on the 3rd day post-seizure. After fixed with $4 \%$ PFA at $4^{\circ} \mathrm{C}$ for $18 \mathrm{~h}$ and embedded using OCT at $-80^{\circ} \mathrm{C}$, samples from the brain and spinal cord were cut into $35 \mu \mathrm{m}$ sections, washed in cold PBS at pH 7.4, blocked with $10 \% \mathrm{BSA}$ at $4^{\circ} \mathrm{C}$ in the dark overnight, and incubated with a primary mouse anti-neuronal nuclei (NeuN) antibody (1:2,000; cat. no. A2050; Sigma-Aldrich; Merck KGaA) at $37^{\circ} \mathrm{C}$ overnight. The sections were washed three times in PBS for 15 min each time and incubated with Alexa Fluor 488-conjugated secondary antibody (1:1,000; cat. no. A-11001; Invitrogen; Thermo Fisher Scientific, Inc.) fofr $3 \mathrm{~h}$ at room temperature. The sections were washed three times with PBS for 5 min each time and mounted using $75 \%$ glycerin. Images were captured using a fluorescent microscope (Scale bars, $500 \mu \mathrm{m}, 100 \mu \mathrm{m}$; Eclipse 80i; Nikon Corporation). ImageJ software (version 1.42q) (National Institutes of Health) was used to quantify the albumin ${ }^{+}$cells in the spinal cord, which allows for the evaluation of the degree of spinal cord injury (10).

Overlap coefficient analysis. To further investigate the relationship between neurons and albumin extravasation, overlap coefficient analysis was conducted using Image-Pro Plus 6.0 (Media Cybernetics, Inc.). Then, two-dimensional curve graphs were constructed to analyze the overlap degree of two fluorescent outputs.

Immunohistochemistry and immunofluorescence analyses. To determine whether leukocyte infiltration occurred in the spinal cord after TLE, immunohistochemistry was conducted to detect the leukocyte adhesion molecule CD11b. In our previous study, leukocyte infiltration was detected in the brain (7). In the present study, immunofluorescence analysis of caspase-3 was conducted to detect apoptosis in the spinal cord following TLE. After samples were fixed with $4 \% \mathrm{PFA}$ at $4^{\circ} \mathrm{C}$ for $18 \mathrm{~h}$ and embedded by $\mathrm{OCT}$ at $-80^{\circ} \mathrm{C}$, sections of the brain and spinal cord were cut into $35 \mu \mathrm{m}$ sections. Slices of spinal cord were blocked with $10 \% \mathrm{BSA}$ at $4^{\circ} \mathrm{C}$ overnight, and incubated with mouse anti-rat CD11b (1:1,000; cat. no. MCA215G; Bio-Rad Laboratories, Inc.) and rabbit anti-rat caspase-3 (1:2,000; cat. no. 96611; Cell Signaling Technology, Inc.) antibodies overnight at $4^{\circ} \mathrm{C}$. For immunohistochemistry, the sections were incubated with a biotinylated-secondary antibody $(1: 3,000$; cat. no. BA9500; Vector Laboratories, Inc.) for $3 \mathrm{~h}$ at room temperature after washing with PBS. Each section was then immersed in color reagent avidin-biotin complex (1:200 each; ABC staining system; cat. no. PK-6100, Vector Laboratories) diluted in PBS for 3 min in the dark, and then mounted with neutral balsam. For immunofluorescence, the sections were incubated with Alexa Fluor 488-conjugated secondary donkey anti-mouse IgG (1:2,000; cat. no. A21202; Invitrogen; Thermo Fisher Scientific, Inc.) overnight at $4^{\circ} \mathrm{C}$, and washed with PBS before mounting with $75 \%$ glycerin. Images were captured using a fluorescent microscope (500 and $100 \mu \mathrm{m}$; Eclipse 80i; Nikon Corporation).

Western blot analysis. Western blotting was performed to detect the expression levels of intercellular adhesion molecule 1 (ICAM-1), CD11b and the inflammatory factors tumor necrosis factor (TNF)- $\alpha$ and interleukin (IL)-6 in the spinal cord. The spinal cord was separated and crushed by an ultrasonic crusher (H150; OUHOR). Then, $600 \mu$ tissue protein lysis buffer (cat. no. R0020; Solarbio Co., Ltd.) was added into $400 \mu \mathrm{l}$ tissue homogenate and placed in ice for $30 \mathrm{~min}$. Supernatant was extracted after the lysates were centrifuged at $8,049 \times \mathrm{g}$ at $4^{\circ} \mathrm{C}$ (Eppendorf centrifuge; 5417C) for $20 \mathrm{~min}$. Protein quantitative analysis was conducted according to the bicinchoninic acid Protein assay kit (cat. no. 23227; Thermo Fisher Scientific, Inc.). In total, $0.1 \mathrm{ml}$ of each unknown sample and BSA standard was placed into separately labeled test tubes and $0.2 \mathrm{ml}$ working reagent (solution $\mathrm{A}$ and $\mathrm{B}$ at 50:1) was added. After mixing, each tube was covered and incubated at $37^{\circ} \mathrm{C}$ for $30 \mathrm{~min}$, and then cooled to room temperature. Measurement of the absorbance of all samples was conducted with the spectrophotometer set to $562 \mathrm{~nm}$ for $10 \mathrm{~min}$. A standard curve was generated by plotting the average blank-corrected $562 \mathrm{~nm}$ measurement for each BSA standard vs. its concentration in $\mu \mathrm{g} / \mathrm{ml}$. Protein concentration of each sample was determined using the standard curve. A total of $50 \mu \mathrm{g}$ protein/lane was separated by $10 \%$ SDS-PAGE and transferred to a PVDF membrane. The membrane was incubated with goat anti-rat ICAM-1 (1:500; cat. no. AF583; R\&D systems, Inc.), mouse anti-rat CD11b (1:800; cat. no. MCA275R; Bio-Rad Laboratories, Inc.), mouse anti-rat TNF- $\alpha$ (1:1,000; cat. no. MAB510; R\&D Systems, Inc.) and goat anti-rat IL-6 (cat. no. AF506; 1:1,500; R\&D Systems, Inc.) antibodies overnight at $4^{\circ} \mathrm{C}$. Mouse monoclonal anti-rat GAPDH (1:1,000; cat. no. sc-365062; Santa Cruz Biotechnology, Inc.) was used to standardize the amount of cytosolic protein. The membrane was then incubated with horseradish peroxidase-conjugated secondary antibody (1:3,000; cat. no. sc-74088; Santa Cruz Biotechnology, Inc.) for $3 \mathrm{~h}$ at room temperature. Diaminobenzidine coloration (DAB staining kit; cat. no. PW017; Sangon Biotech Co., Ltd.) was conducted at $37^{\circ} \mathrm{C}$ in the dark for $1 \mathrm{~min}$, and the Tanon 4200 imaging system (Tanon Science and Technology Co., Ltd.) was used to observe the results. Analysis was conducted using ImageJ software (version 1.42q; National Institutes of Health). 
Fluoro-Jade $C(F J C)$ staining. After washing with PBS at a $\mathrm{pH} 7.4$, the brain and spinal cord sections $(35 \mu \mathrm{m})$ were incubated in 0.3 M Triton X-100 and 0.1 M DAPI solution at room temperature for $2 \mathrm{~h}$. The sections were washed with PBS and dried at $50^{\circ} \mathrm{C}$ for $1 \mathrm{~h}$, before buffering in $99.9 \%$ alcohol and then $70 \%$ alcohol at room temperature for 5 min each. Sections were washed twice by immersion in distilled water for $10 \mathrm{~min}$ each. The sections were then submerged in $0.06 \%$ potassium permanganate solution at room temperature for $10 \mathrm{~min}$ and gently agitated. After rinsing three times in distilled water, the sections were placed in $0.01 \%$ FJC working solution (EMD Millipore) at $4^{\circ} \mathrm{C}$ in the dark for $20 \mathrm{~min}$, washed in distilled water and dried in an oven at $50^{\circ} \mathrm{C}$ for $10 \mathrm{~min}$. All sections were mounted with $60 \%$ neutral balsam and left to air-dry for $\geq 1 \mathrm{~min}$.

Statistical analysis. Data were analyzed using SPSS 24.0 (IBM Corp.) and are presented as the mean \pm SEM. ANOVA test was used to compare the mean values. Dunnett's test was used for comparison between experimental group and control group. Tukey's test was used in pairwise comparison among three groups. Rank-sum test was used to conduct behavioral analyses. $\mathrm{P}<0.05$ was considered to indicate a statistically significant difference.

\section{Results}

Baseline analysis. The body weights of the rats did not differ significantly between groups (Table I; $\mathrm{P}=0.725$ ). All rats were raised in the same conditions and no drugs were administration prior to experimentation.

Behavioral analysis and EEG recording. Behavioral analysis results identified grade II-V seizures in both the KA and the PL group (Table II). The present results suggested that rats in the control group exhibited normal brain activity (Fig. 1A), while sharp and slow waves were recorded in the PL group during upper limb convulsion (Fig. 1B). In addition, long-lasting multiple-spike waves were observed in the KA group during tonic-clonic seizures (Fig. 1C).

Tissue damage in the brain and spinal cord following seizure. TEM results suggested that the structure of the microvessels (Fig. 2A) and myelin sheath (Fig. 2E) were normal in the control group. However, significant edema and vacuolization of the BBB basement membrane were observed in the brain (Fig. 2B) and spinal cords (Fig. 2C) of rats in the KA group. Separation of the basement membrane was detected in the spinal cords of the PL group (Fig. 2D). The present study identified significant separation of the myelin sheath in the brain (Fig. 2F) and spinal cords (Fig. 2G) of the KA group, while a lesser degree of separation was observed in the spinal cords of the PL group rats (Fig. 2H). Brain-water content analysis results suggested a significant brain edema in both the KA ( $\mathrm{P}=0.006)$ and the PL group $(\mathrm{P}=0.031)$ compared with the control group. However, the degree of spinal cord edema compared between control group and TLE groups (both KA and PL groups) was not significant (Fig. 2I). The present results suggested that basal lamina separation of the BBB and structural looseness of the myelin sheath were
Table I. Baseline weights of male Sprague-Dawley rats in all groups. No significant difference was observed between groups at the baseline level.

\begin{tabular}{lc}
\hline Group & Weight, $\mathrm{g}$ \\
\hline Control group (total, $\mathrm{n}=30)$ & $270.58 \pm 2.12$ \\
PL group ( $\mathrm{n}=30)$ & $273.02 \pm 2.53$ \\
KA group ( $\mathrm{n}=30)$ & $269.33 \pm 2.05$ \\
P-value (total, ANOVA) & 0.725 \\
\hline
\end{tabular}

PL, pilocarpine; KA, kainic acid.

Table II. Behavioral analysis of rats in all groups.

\begin{tabular}{lrrrrrrr}
\hline & \multicolumn{7}{c}{ Grade } \\
\cline { 2 - 6 } Groups & 0 & I & II & III & IV & V & Death \\
\hline Control (total, $n=30)$ & 30 & 0 & 0 & 0 & 0 & 0 & 0 \\
KA (n=30) & 0 & 0 & 3 & 5 & 14 & 8 & 0 \\
PL (n=30) & 0 & 0 & 1 & 8 & 11 & 10 & 0 \\
\hline
\end{tabular}

PL, pilocarpine; KA, kainic acid. Control vs. KA, $\mathrm{P}<0.001$; Control vs. PL, $\mathrm{P}<0.001$; KA vs. PL, $\mathrm{P}=0.834$.

predominantly observed in the spinal cords of rats with grade IV (8/25)-V (12/18) seizure attacks.

Neuronal cell damages in the brain and spinal cord following seizure. Nissl staining results indicated severe structural damage and decreased neuronal cell numbers in the brains of the PL group (Fig. 3B and D; $\mathrm{P}<0.001$ ) and the KA group (Fig. 3C and D; $\mathrm{P}<0.001$ ), compared with normal staining in the control group (Fig. 3A). Semi-quantitative analysis results suggested a significant decrease in the gray value of the Nissl bodies in the spinal cords of both the PL and KA group (Fig. 3D; $\mathrm{P}<0.001)$. The mean area of $\mathrm{Niss}^{+}$cells in the spinal cord was significantly decreased in the KA group (Fig. 3G and H; $\mathrm{P}=0.041$ ) compared with control group (Fig. $3 \mathrm{E}$ and $\mathrm{H}$ ). The mean area of $\mathrm{Niss}^{+}$cells was slightly decreased in the PL group (Fig. 3F) compared with control group (Fig. 3E), but the difference was not statistically significant (Fig. 3H; $\mathrm{P}=0.773)$.

Albumin extravasation in the brain and spinal cord following seizure. Albumin extravasation was not detected in the brain (Fig. 4A) or the spinal cords (Fig. 4C) of the control group. However, significant albumin extravasation was observed in the brain (Fig. 4B) and the spinal cord (Fig. 4D and I) in both the PL and KA groups, particularly in the hippocampus (Fig. 4B), cervical cord (Fig. 4D, G and J) and thoracic cord (Fig. 4E, $\mathrm{H}$ and J). A lesser degree of albumin extravasation was observed in the lumbar cord (Fig. 4F, I and J). The present results suggested that in the spinal cord albumin extravasation was primarily observed in rats with grade IV-V seizures. Additionally, double-staining results identified the presence of 

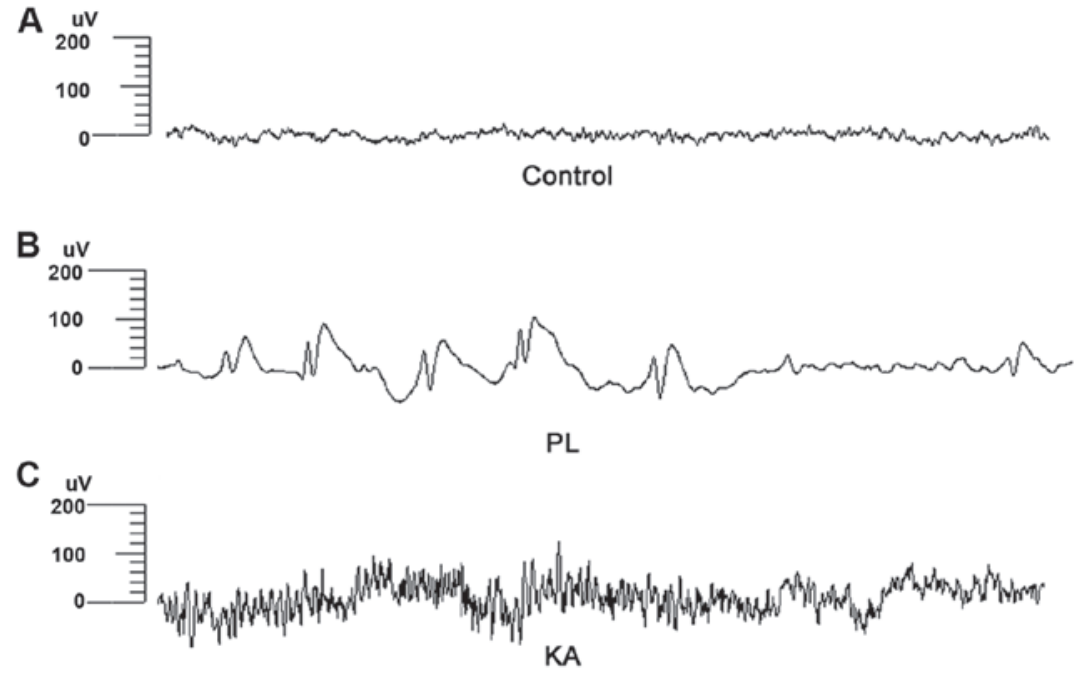

Figure 1. Electroencephalograph recordings. (A) Rats in the control group showed normal brain activity. (B) A sharp and slow wave rhythm was observed in the PL group during myoclonic seizure attacks. (C) Multi-spike waves were recorded in the KA group during tonic-clonic seizure attacks. PL, pilocarpine; KA, kainic acid.

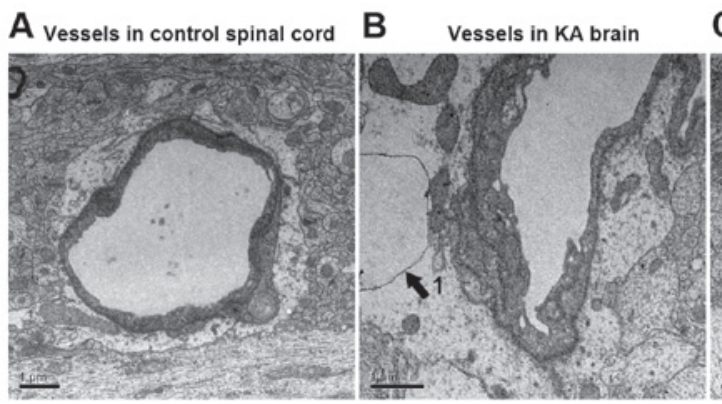

C Vessels in KA spinal cord

D Vessels in PL spinal cord
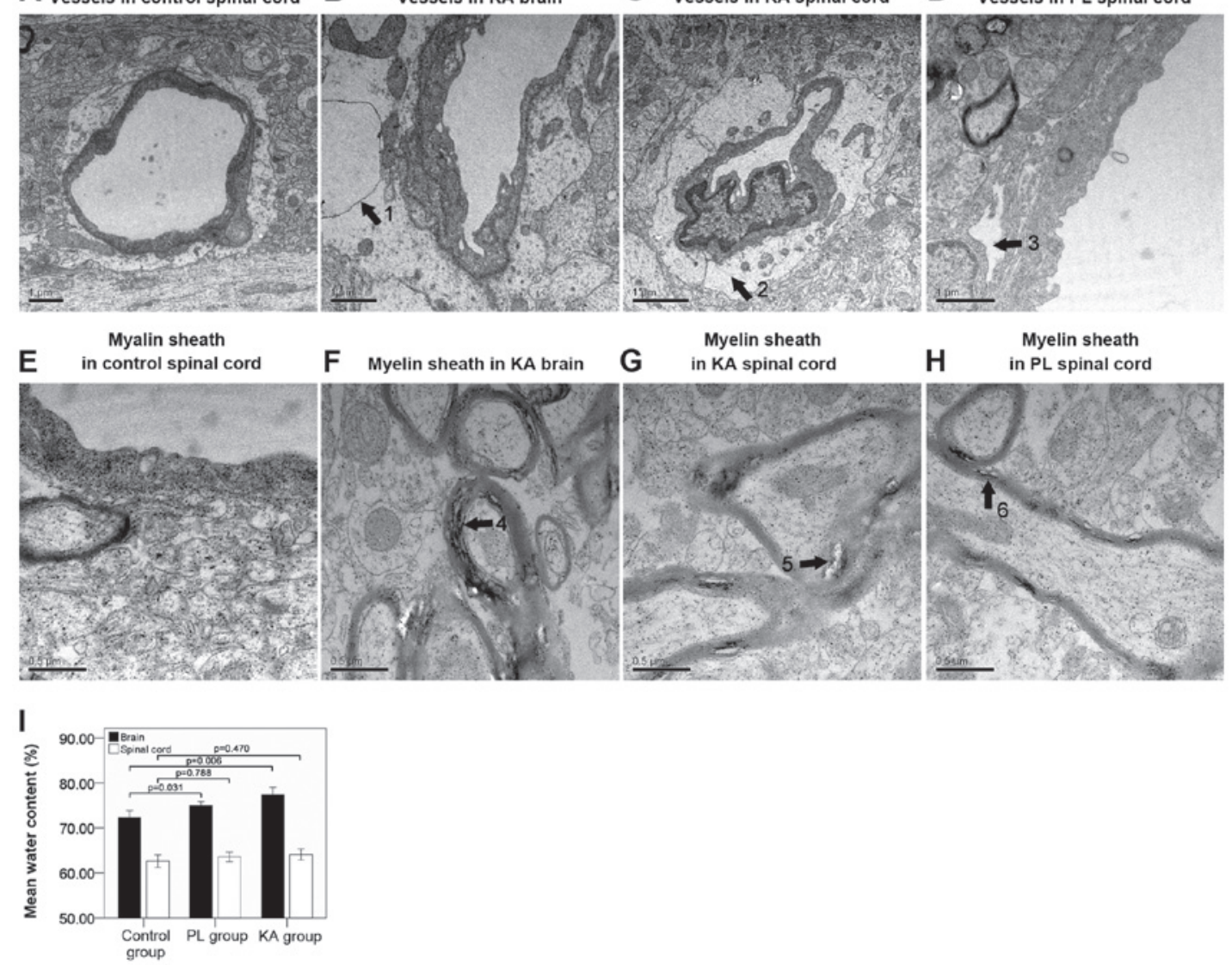

Figure 2. Tissue damage following seizure shown by transmission electron microscopy. (A) Normal vessel structure in the control group. Edema and vacuolization of the basement membrane in the (B) brain and (C) spinal cord in the KA group. (D) Separation of the basement membrane in the spinal cords of the PL group. Scale bar, $1 \mu \mathrm{m}$. (E) Normal myelin sheath structure in the control group. Significant structural looseness and separation of the myelin sheath in the (F) brain and (G) spinal cord in the KA group. (H) Structural looseness of the myelin sheath in the spinal cords of the PL group. Scale bar, $0.5 \mu \mathrm{m}$. (I) Analysis of the brain water content showed different degrees of edema in the brain and spinal cord in the KA and the PL group. Arrow one, vacuolization. Arrow two, edema of the basement membrane. Arrow three, separation of the basement membrane. Arrows 4-6, looseness and separation of the myelin sheath. PL, pilocarpine; KA, kainic acid.

albumin in the neuronal cells (Fig. 4K-M). This colocalization effect of albumin in neuronal cells was also identified by overlap analysis results (Fig. 4N), which is in line with results from previous studies (7). 

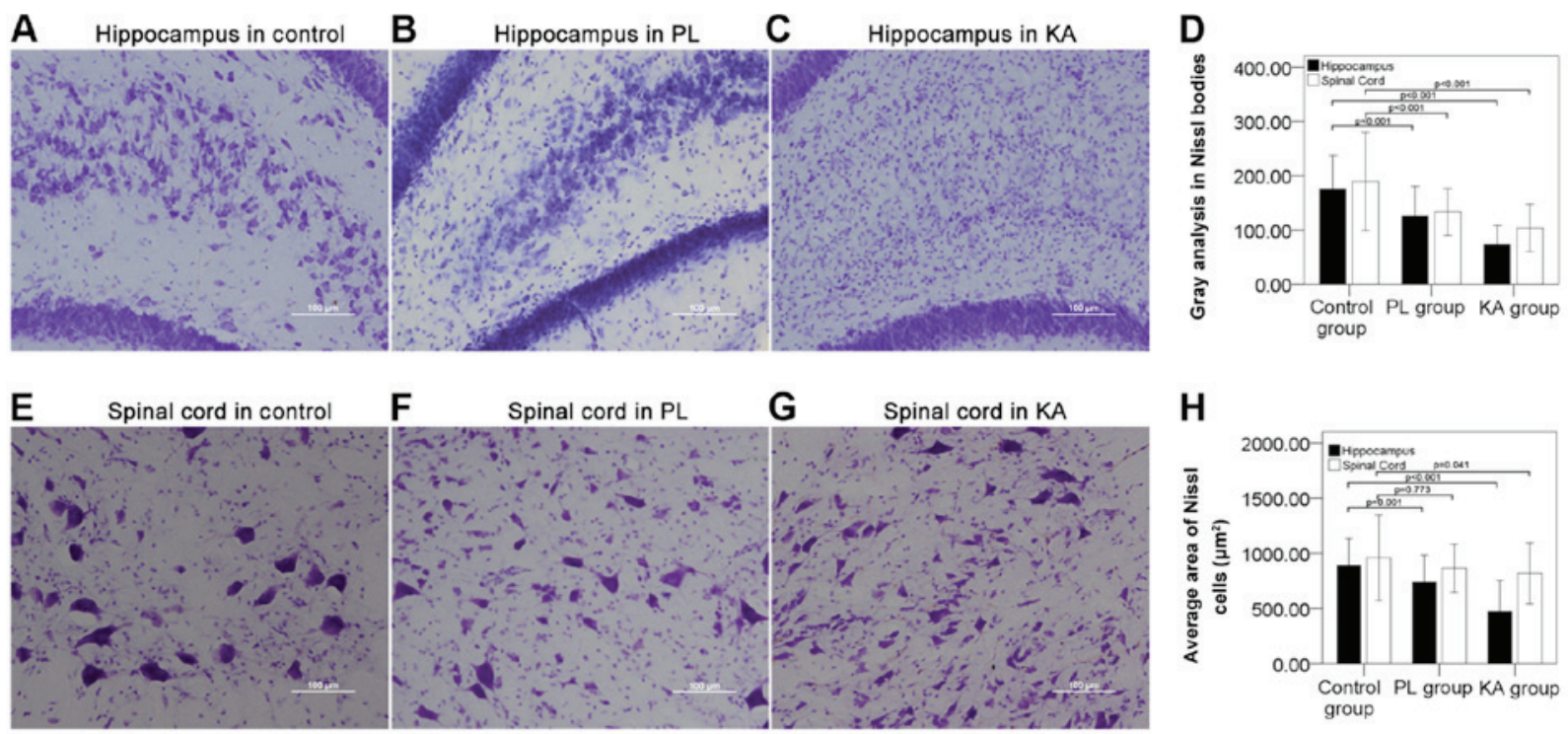

Figure 3. Nissl staining. (A) Normal structure of the hippocampus in the control group. Significant structural changes and neuronal cell loss in the hippocampi of the (B) PL and (C) KA groups. (D) Gray value decreased in Nissl ${ }^{+}$cells of the hippocampus and spinal cord in the PL and KA groups. (E) Normal structure of the spinal cord in the control group. Cell volume decreased and morphological changes of Nissl ${ }^{+}$cells in the spinal cord in the (F) PL and (G) KA groups. (H) Mean area of $\mathrm{Niss}^{+}$cells in the hippocampus decreased in both groups, and in the spinal cord in the KA group only. Scale bar, $100 \mu \mathrm{m}$. PL, pilocarpine; KA, kainic acid .

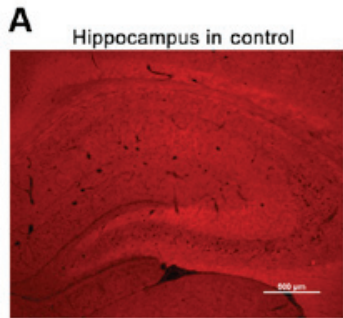

E

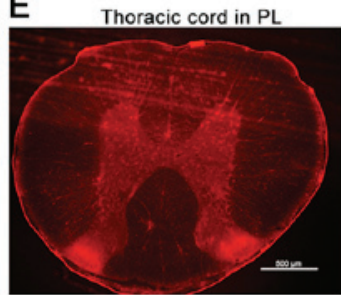

I
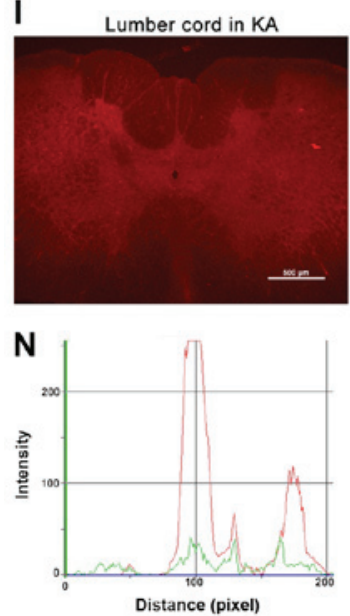

B

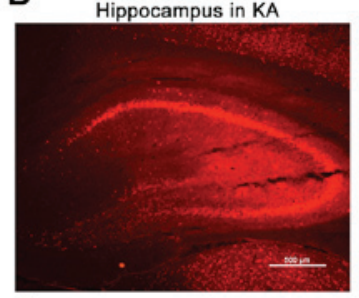

F

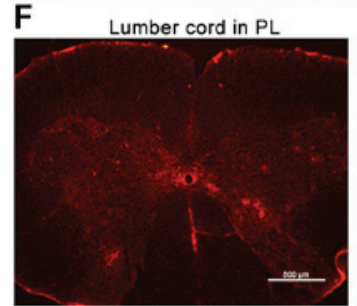

J

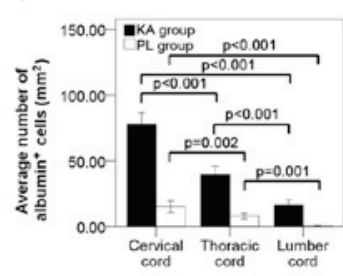

C

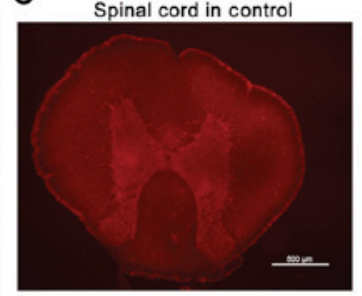

G

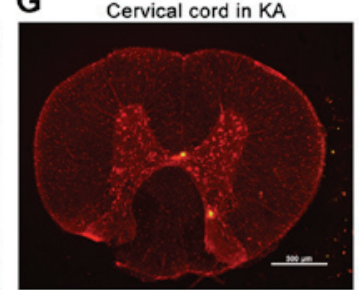

K

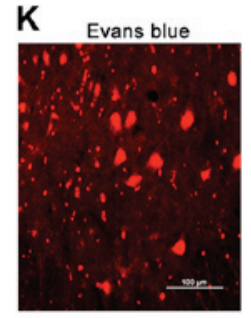

L
D

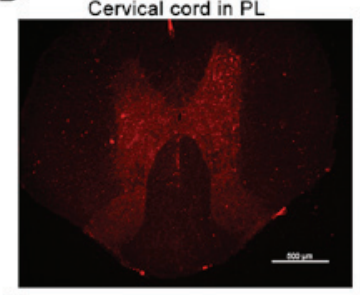

H
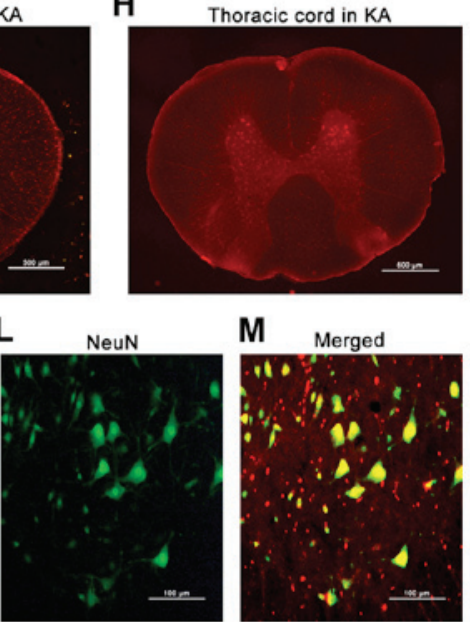

M Merged

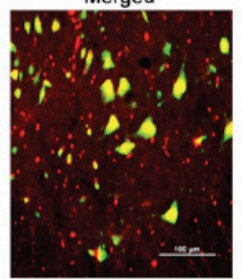

Figure 4. Albumin and NeuN staining. Albumin was not detected in the (A) hippocampus of the control group. (B) Significant albumin staining was detected in the hippocampus in the KA group. Albumin was not detected in the (C) spinal cord in the control group, but significant albumin staining was detected in (D) cervical, (E) thoracic and (F) lumbar cord in the PL group. In addition, significant albumin staining was detected in the (G) cervical, (H) thoracic and (I) lumbar cord in the KA group. Scale bar, $500 \mu \mathrm{m}$. (J) Quantification of albumin staining in KA and PL groups. A low degree of albumin staining was observed in the lumbar cord. Double-staining (M) of albumin (K) and neuronal cells (L) showed significant match (N). Scale bar, $100 \mu \mathrm{m}$. PL, pilocarpine; KA, kainic acid; NeuN, neuronal nuclei. 

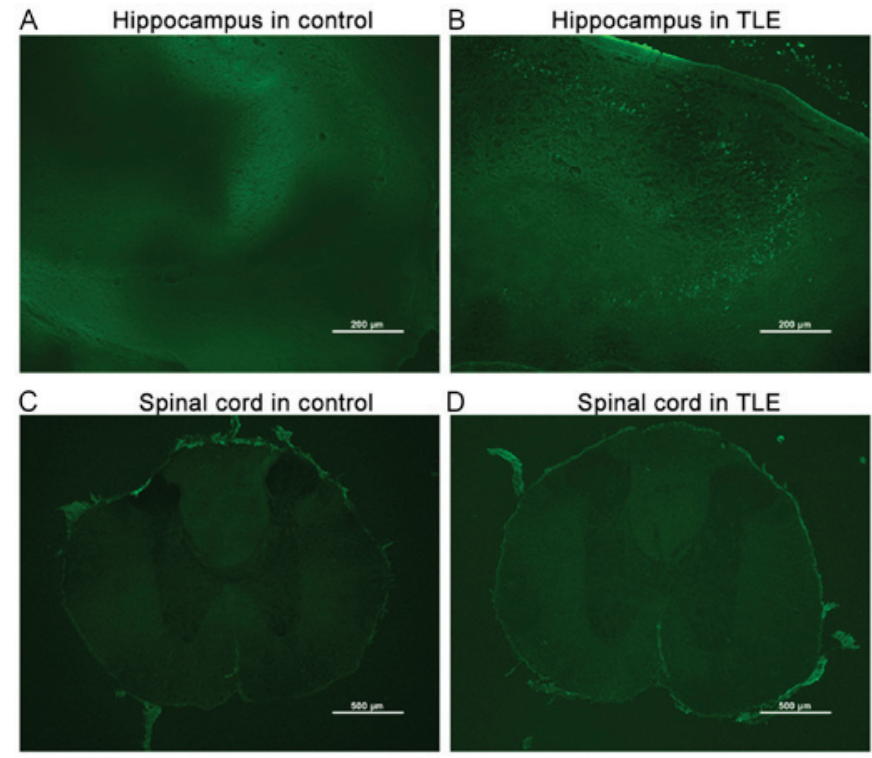

Figure 5. FJC staining. (A) No significant difference was observed in the hippocampus in the control group. (B) Neuronal degeneration was observed in the hippocampus in the TLE group. Scale bar, $200 \mu \mathrm{m}$ (C) No significant difference was observed in the spinal cord in the control group. (D) No signals were detected in the spinal cords of the TLE group. Scale bar, $500 \mu \mathrm{m}$. Arrow, FJC signals. FJC, Fluoro-Jade C; TLE, temporal lobe epilepsy.

Neuronal degeneration in the brain, but not the spinal cord following seizure. The present results suggested that $\mathrm{FJC}^{+}$ staining was not detected in the brain (Fig. 5A) or spinal cords (Fig. 5C) of the control group. The present results suggested that a small degree of $\mathrm{FJC}^{+}$staining was detected in the cortex and hippocampus (Fig. 5B), but not in the spinal cords (Fig. 5D) of the TLE group.

Increases in ICAM-1 expression level in the spinal cord after $T L E$. To investigate the involvement of BBB injury in the spinal cord after TLE, the expression level of the vascular adhesion molecule ICAM-1 was assessed using immunofluorescence and western blotting. The present results suggested that following TLE, the expression levels of ICAM-1 were significantly increased in the injured spinal cords in both the PL group (Fig. 6B, D and E; $\mathrm{P}=0.007$ ) and the KA group (Fig. 6C-E; $\mathrm{P}=0.002$ ), compared with the control group (Fig. 6A).

Increases in the expression levels of inflammatory factors in the spinal cord after TLE. To investigate whether an inflammatory reaction contributed to spinal cord injury following TLE, the expression levels of the leukocyte adhesion molecule CD11b, which binds to the receptor ICAM-1, and TNF- $\alpha$ and IL- 6 were detected using immunohistochemistry and western blotting. The present results suggested that a small number of CD11 $\mathrm{b}^{+}$ leukocytes had infiltrated into the spinal cord in the PL group (Fig. 7B and D) and the KA group (Fig. 7C and D) compared with the control group (Fig. 7A). Furthermore, western blot analysis results suggested a significant increase in TNF- $\alpha$ and IL-6 expression levels in both the KA and PL groups (Fig. 7E-H) compared with the control group.

Apoptosis in the spinal cord after TLE. To investigate the role of apoptosis in spinal cord injury following TLE, the expression level of caspase-3 was detected using immunofluorescence analysis. Compared with the control group (Fig. 8A), a significant increase in the number of caspase $-3^{+}$cells was detected in the spinal cord after TLE in the PL (Fig. 8B and D) and KA groups (Fig. 8C and D). Multiple-staining of caspase-3 (Fig. 8E), albumin (Fig. 8F) and nucleus (Fig. 8G) revealed caspase- $3^{+}$staining in albumin ${ }^{+}$neurons (Fig. $8 \mathrm{H}$ ).

\section{Discussion}

Spinal cord injury following epileptic seizure has been reported in a number of clinical cases (13). However, whether spinal cord injury frequently occurs following seizure and its associated pathological characteristics have not, to the best of our knowledge, previously been investigated.

The present results suggested that following TLE, the number and volume of Nissl bodies was significantly decreased, thus indicating neuronal damage. Structural looseness and separation of the myelin sheath were also identified in the present study, which are also suggestive of neuronal axon injury. The present results suggested that pathological changes were observed in both the brain and the spinal cord, but were more significant in the hippocampus, cervical cord and thoracic cord. To further investigate the possible mechanism of spinal cord injury, the integrity of the BBB and indicators of the inflammatory response were assessed; our previous study indicated that significant BBB damage was associated with neuronal dysfunction (10). The present TEM results suggested a significant vacuolization and edema of the BBB basement membrane. In addition, significant serum albumin extravasation into the brain and the spinal cord was also detected, further indicating structural and functional damage to the BBB as a result of spinal cord injury. Double-staining results suggested that albumin was localized to the neurons, indicating that neuronal damage may be associated with albumin extravasation and excessive albumin absorption, which is in line with results from a previous study (10). In the present study, neuronal degeneration was not detected in the spinal cord, but a small number of albumin ${ }^{+}$ neurons stained positive for caspase-3, which is an indicator of apoptosis (14). Therefore, the present results require further investigation.

To investigate the involvement of the inflammatory response in spinal cord injury, leukocyte infiltration and the expression levels of inflammatory mediators were assessed in the present study. Western blot analysis results suggested a significant elevation in the expression levels of the leukocyte adhesion molecule CD11b and the receptor for CD11b ICAM-1, which mediates leukocyte infiltration into the brain (15). The present immunohistochemistry results also indicated a small number of $\mathrm{CD}_{11 b^{+}}$leukocytes in the spinal cord. Moreover, western blotting results suggested a significant increase in the expression levels of TNF- $\alpha$ and IL-6, which was indicative of an inflammatory reaction during seizure-associated spinal cord injury.

Case reports have supported the hypothesis that spinal cord injury is primarily caused by trauma, as head injury, spinal canal stenosis and vertebral fracture are observed in a number of patients, particularly walking adults with a high incidence of fall accidents $(2,3)$. However, not all patients with spinal cord 
A

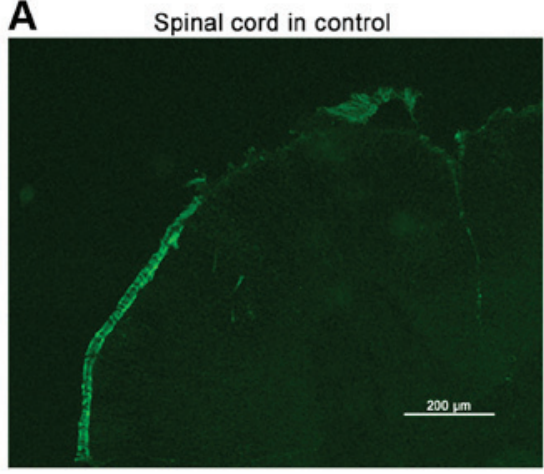

D

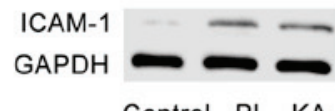

Control PL KA
B

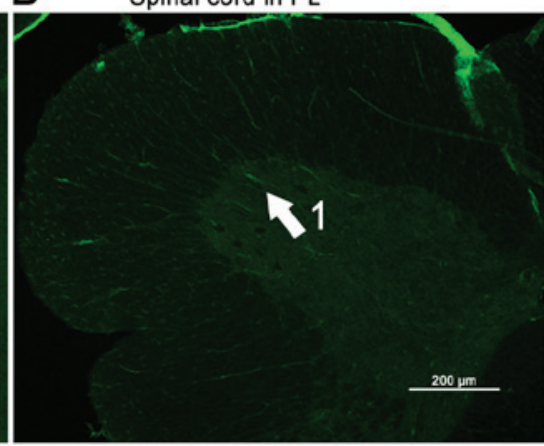

E

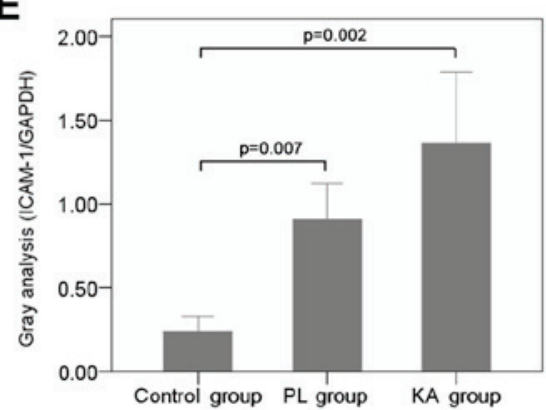

C Spinal cord in KA

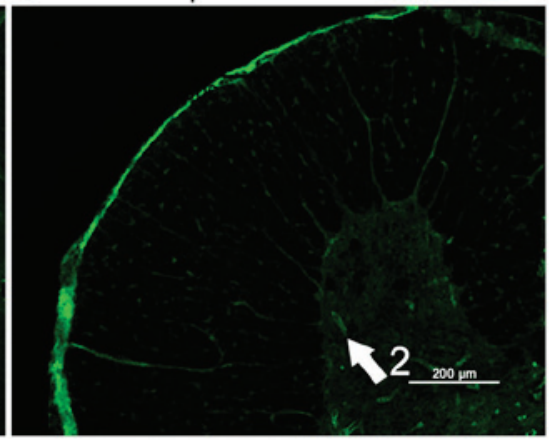

Figure 6. ICAM-1 expression in the spinal cord. (A) Expression level of the vascular adhesion molecule ICAM-1 in the control group. Significant expression level of ICAM-1 along the blood vessels in the (B) PL and (C) KA groups. Scale bar, $200 \mu$ m. (D) Western blotting results suggested a significant increase in ICAM-1 expression level in the spinal cord in the PL and KA groups. (E) Quantification of western blotting results. Arrow, ICAM-1 expression along the vessels. ICAM-1, intercellular adhesion molecule 1; PL, pilocarpine; KA, kainic acid.
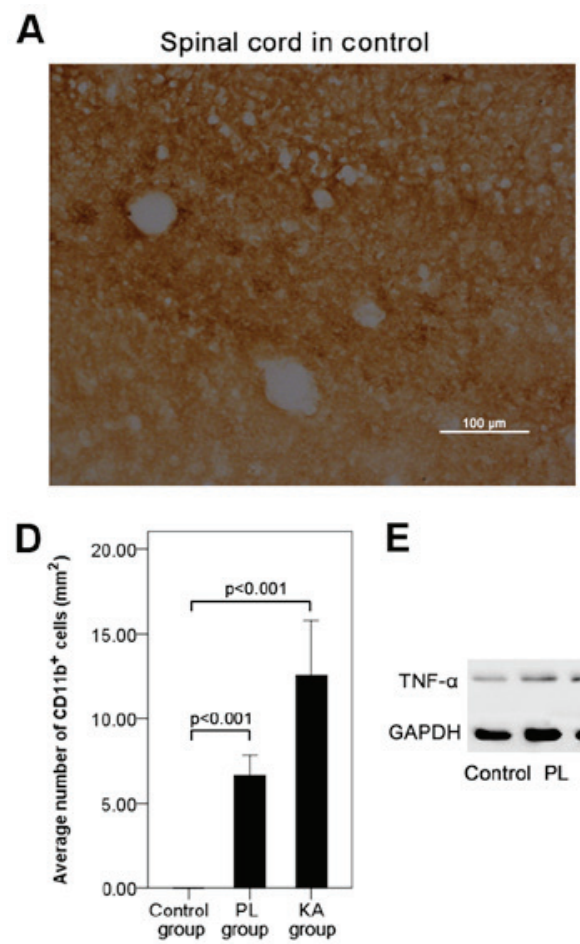

E
B
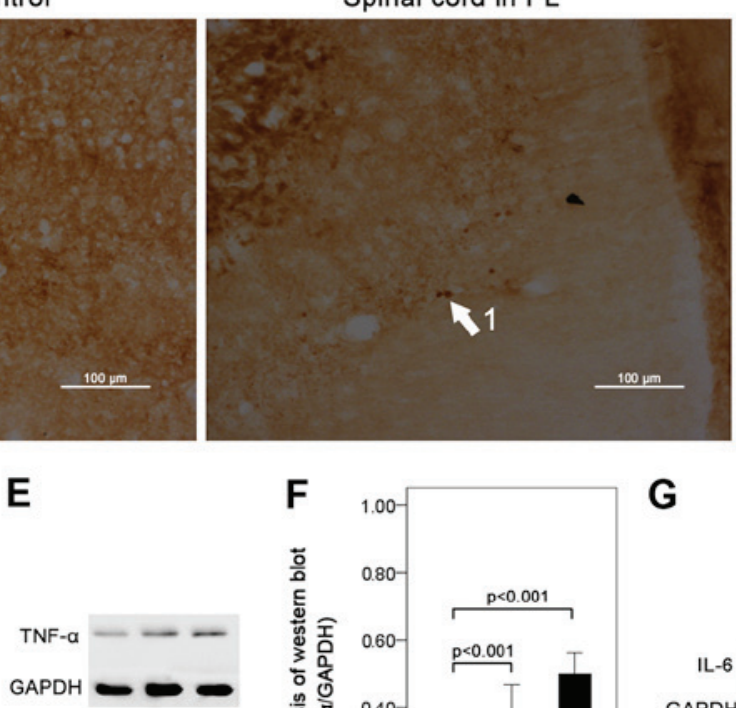

Control PL KA

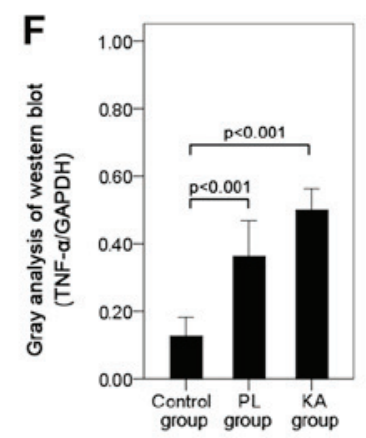

C Spinal cord in KA

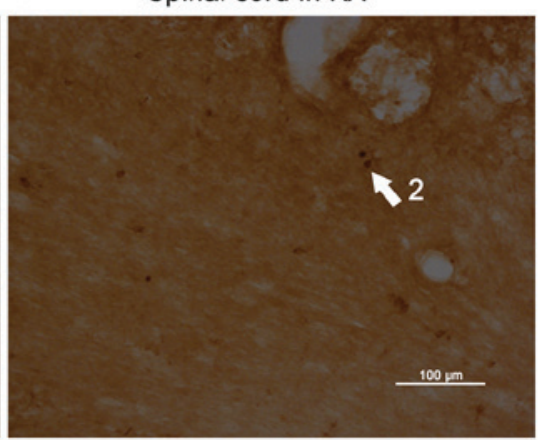

G
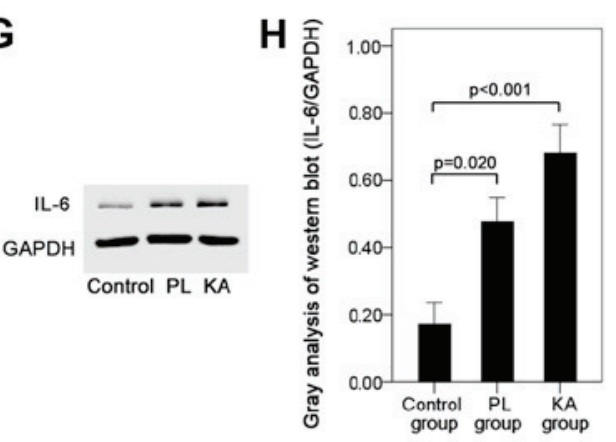

Figure 7. Expression levels of CD11b and inflammatory factors. (A) Leukocyte adhesion molecule CD11b was not detected in the spinal cord in the control group. A small number of CD11b+ leukocytes were detected in the spinal cord in the (B) PL group and (C) the KA group. (D) Quantification of the average number of $\mathrm{CD}_{11 b^{+}}$cells. (E) Western blotting results suggested that expression levels of (F) TNF- $\alpha$ were significantly increased in the spinal cord in the PL and KA groups. (G) Western blotting results suggested that expression levels of (H) IL-6 were significantly increased in the spinal cord in the PL and KA groups. Arrow one and two, CD11b leukocytes. PL, pilocarpine; KA, kainic acid; TNF- $\alpha$, tumor necrosis factor $\alpha$; IL-6, interleukin-6.

injury present with evidence of trauma or other potential injuries (1), indicating that there are other mechanisms of spinal cord injury after seizure. The present results suggested that pathological changes were primarily detected in rats that had 

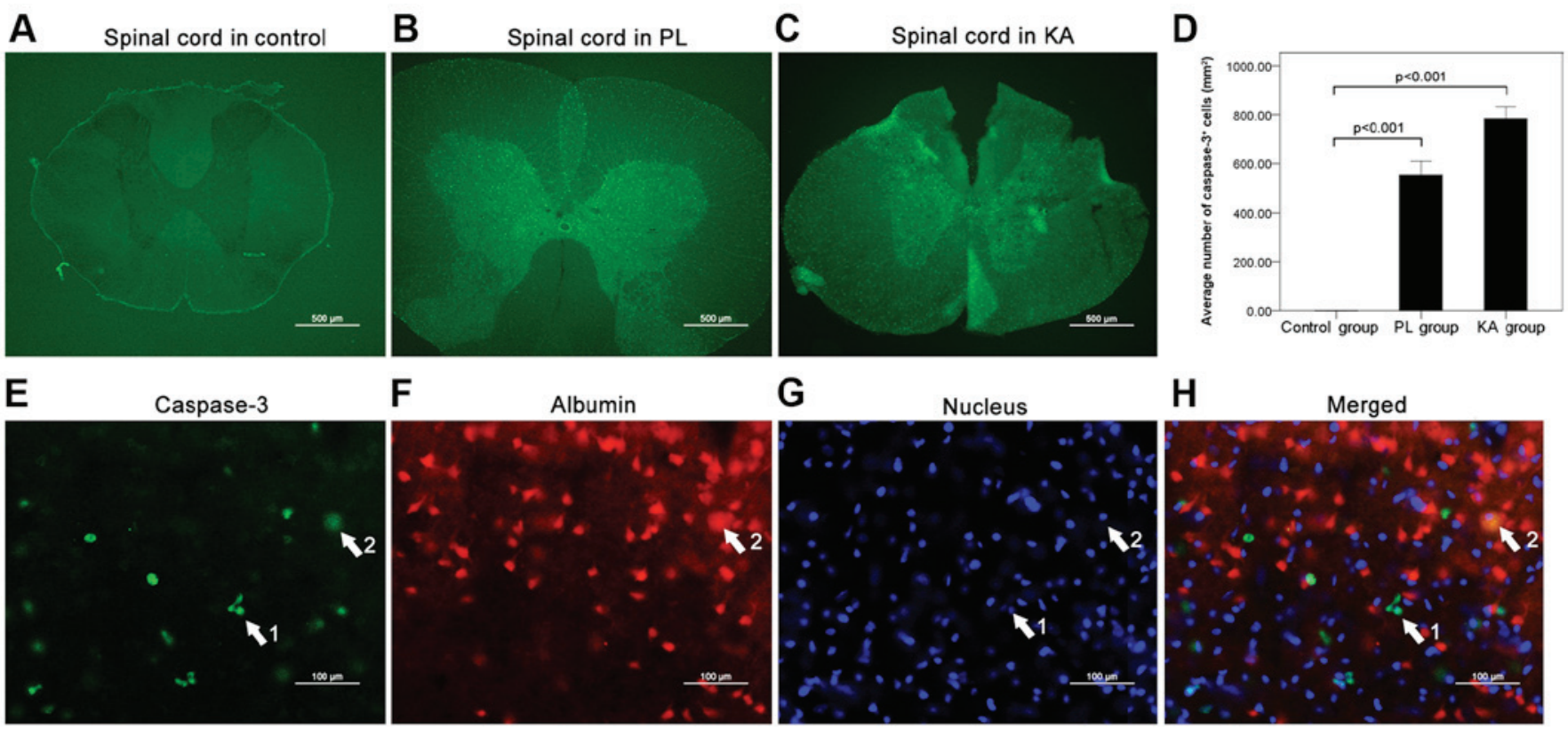

Figure 8. Apoptosis in the spinal cord following TLE. (A) Caspase-3 was not detected in the control group spinal cord tissues. Increased expression levels of caspase-3 were observed in the spinal cord after TLE in the (B) PL and (C) KA groups. Scale bar, $500 \mu \mathrm{m}$. (D) Quantification of the average number of casapase- $3^{+}$cells. (H) Multiple-staining (E) of caspase-3, (F) albumin ad the (G) nucleus indicated a small degree of caspase-3 staining in albumin ${ }^{+}$cells. Arrow one, merged caspase-3 and nuclei staining. Arrow two, merged caspase-3, albumin and nuclei staining. TLE, temporal lobe epilepsy; PL, pilocarpine; KA, kainic acid.

experienced grade IV and V seizures. Furthermore, the greatest degree of spinal cord injury was observed in the cervical and thoracic cord, which is consistent with previous clinical reports $(2,3)$. However, trauma-associated spinal cord injury is not uniformly distributed within the spinal cross-section and most of the injuries are accompanied by edema (16). In addition, the extent of spinal cord damage is dependent on the degree of vascular injury (16). The present results suggested that the pathological change in the spinal cross-section had bilateral symmetry and macroscopic structural damage was not observed. The present results suggested that neuronal damage was predominantly distributed in the anterior horn of the spinal cord, and grade IV seizure was not accompanied by trauma or falls. The present results do not support the view that spinal cord injury is caused by trauma, thus further studies are required for clarification of these results.

Systemic inflammatory responses occur during epileptogenesis (6). The present study identified an inflammatory reaction in the PL and KA groups, however pathological changes in the spinal cord were largely located in the cervical and the thoracic cord, which does not support the previously reported mechanism of systemic inflammation (6). In the current study, upper limb convulsions were indicated to be a frequent behavioral manifestation of rats during seizure attacks. Injured neurons are primarily distributed in the anterior horn in the spinal cord. If spinal cord injury was associated with hyperactivity of the spinal neurons remains to be determined. Moreover, the brain and spinal cord are closely associated, possessing similar neurotransmitter expression profiles (8) and the brain is theorized to originate from the spinal cord (7). The principal cause and potential mechanism of spinal cord injury following seizure remains to be elucidated, and the potential sequela and reversible nature of these injuries requires further investigation.
In conclusion, the present results suggested that spinal cord injury may commonly occur in rats following severe seizure attacks. The present results suggested that seizures caused damage to the BBB, albumin extravasation, inflammation and apoptosis, which was associated with spinal cord injury and its pathological changes.

\section{Acknowledgements}

The authors would like to thank Professor YuQiang Ding and his colleagues affiliated to the Department of neuropathophysiology, Tongji University, Shanghai, for their technical and theoretical support.

\section{Funding}

The present study was supported by a grant from the National Natural Science Foundation of China (grant no. 81271441).

\section{Availability of data and materials}

The datasets used and/or analyzed during the current study are available from the corresponding author on reasonable request.

\section{Author's contributions}

$\mathrm{JL}, \mathrm{SL}$ and $\mathrm{YZ}$ designed the main content and protocol of this study. The experiments were conducted by JL and SL. Supplementary experiments were conducted by ZL. Data collection and statistical analysis were conducted by GL and KG. HZ edited the figures. HZ, HW ZL and LZ participated and gave important advice in the design and redesign of the study, and also aided in performing the study, including 
improvement of test procedure, image analysis and revision of the article.

\section{Ethics approval and consent to participate}

The present study was approved by The Ethics Committee of The First People's Hospital of Shanghai Jiaotong University (approval no. 2012-DF-50).

\section{Patient consent for publication}

Not applicable.

\section{Competing interests}

The authors declare that they have no competing interests.

\section{References}

1. Lee S, Lee JE, Yang S and Chang H: A case of central cord syndrome related status epilepticus-a case report. Ann Rehabil Med 35: 574-578, 2011

2. Roohi F and Fox A: Burst fracture of the first lumbar vertebra and conus-cauda syndrome complicating a single convulsive seizure: A challenge of diagnosis in the emergency department J Emerg Med 31: 381-385, 2006.

3. Kruitbosch JM, Schouten EJ, Tan IY, Veendrick-Meekes MJ and de Vocht JW: Cervical spinal cord injuries in patients with refractory epilepsy. Seizure 15: 633-636, 2006.

4. Zwimpfer TJ, Brown J, Sullivan I and Moulton RJ: Head injuries due to falls caused by seizures: A group at high risk for traumatic intracranial hematomas. J Neurosurg 86: 433-437, 1997.

5. Fukuhara N: Fukuhara disease. Brain Nerve 60: 53-58, 2008 (In Japanese).
6. Gouveia TL, Vieira de Sousa PV, de Almeida SS, Nejm MB, Vieira de Brito JM, Cysneiros RM, de Brito MV, Salu BR, Oliva ML, Scorza FA and Naffah-Mazzacoratti Mda G: High serum levels of proinflammatory markers during epileptogenesis. Can omega-3 fatty acid administration reduce this process? Epilepsy Behav 51: 300-305, 2015.

7. Riva MA, Bellani I, Tremolizzo L, Lorusso L, Ferrarese C and Cesana G: The neurologist in dante's inferno. Eur Neurol 73: 278-282, 2015

8. Shen HY, van Vliet EA, Bright KA, Hanthorn M, Lytle NK, Gorter J, Aronica E and Boison D: Glycine transporter 1 is a target for the treatment of epilepsy. Neuropharmacology 99: 554-565, 2015.

9. Racine RJ: Modification of seizure activity by electrical stimulation. II. Motor seizure. Electroencephalogr Clin Neurophysiol 32 281-294, 1972.

10. Liu Z, Liu J, Wang S, Liu S and Zhao Y: Neuronal uptake of serum albumin is associated with neuron damage during the development of epilepsy. Exp Ther Med 12: 695-701, 2016.

11. Liu Z, Wang S, Liu J, Wang F, Liu Y and Zhao Y: Leukocyte infiltration triggers seizure recurrence in a rat model of temporal lobe epilepsy. Exp Ther Med 12: 695-701, 2016.

12. Jungner M, Grände PO, Mattiasson G and Bentzer P: Effects on brain edema of crystalloid and albumin fluid resuscitation after brain trauma and hemorrhage in the rat. Anesthesiology 112: 1194-1203, 2010.

13. Cottrell P, Ahmed S, James C, Hodson J, McDonnell PJ, Rauz S and Williams GP: Neuron J is a rapid and reliable open source tool for evaluating corneal nerve density in herpes simplex keratitis. Invest Ophthalmol Vis Sci 55: 7312-7320, 2014.

14. Brentnall M, Rodriguez-Menacol L, De Guevara RL, Cepero E and Boise LH: Caspase-9, caspase-3 and caspase-7 have distinct roles during intrinsic apoptosis. BMC Cell Biol 14: 32, 2013.

15. Rahman A and Fazal F: Hug tightly and say goodbye: Role of endothelial ICAM-1 in leukocyte transmigration. Antioxid Redox Signal 11: 823-839, 2009.

16. Perovitch M, Perl S and Wang H: Current advances in magnetic resonance imaging (MRI) in spinal cord trauma: Review article. Paraplegia 30: 305-316, 1992

(i) $\Theta$ This work is licensed under a Creative Commons Attribution-NonCommercial-NoDerivatives 4.0 International (CC BY-NC-ND 4.0) License. 